\title{
Retraction
}

\section{Retraction: Hires et al, Whisker Dynamics Underlying Tactile Exploration}

At the request of the authors, The Journal of Neuroscience is retracting "Whisker Dynamics Underlying Tactile Exploration" by S. Andrew Hires, Alexander L. Efros, and Karel Svoboda, which appeared on pages 9576-9591 of the June 5, 2013 issue. The authors report, "After publication we discovered that higher-order eigenmodes were incorrectly summed when calculating the timedependence of whisker shape during touch with a rigid object. Correction of this error revealed that our boundary conditions were inappropriate for the whisker-object interactions treated in our paper. Modification of these boundary conditions will alter the results presented in Figures 6-11. We therefore wish to withdraw the article. A corrected treatment will be published in the future. We apologize for any confusion caused by this error."

DOI: 10.1523/JNEUROSCI.3625-13.2013 